\title{
Application of Intuitionistic Fuzzy Evaluation Method in Aircraft Cockpit Display Ergonomics
}

\author{
Hui Liu, Chengli Sun, and Jiliang Tu \\ School of Information and Engineering, University of Nanchang Hangkong, China
}

\begin{abstract}
The ergonomic level of cockpit display design can be improved by establishing an objective and effective method for evaluating the ergonomics of the cockpit display. Given the fuzz problem in ergonomic evaluation, a new intuitionistic fuzzy evaluation method is proposed based on the Intuitionistic Fuzzy Ordered Weighted Geometric Average (IFOWGA) operator and the possible degree function in this work. Firstly, the intuitionistic fuzzy evaluation matrix considering the hesitation degree of experts' determination is first constructed as the basis of intuitionistic fuzzy evaluation. Secondly, using the IFOWGA operator the intuitionistic fuzzy evaluation values are obtained through aggregating the evaluation matrix. And these values are ranked to get the level of ergonomic evaluation by possible degree ranking function. Finally, an evaluation example based on the cockpit display of a certain aircraft is given to verify the effectiveness of the proposed approach. Six alternatives of the evaluation result are obtained by the aggregation of the IFOWGA operator. Applied the possible degree function, the ergonomic evaluation grade of the aircraft cockpit display is the second level by ranking the alternative sand the variation of intuitionistic fuzzy value is already small when the number of experts is more than 16. It can be shown from the results that the ergonomic level of cockpit display can be objectively and scientifically evaluated by the proposed quantitative method, and it can provide a theoretical basis and practical methods for improving the ergonomic level of cockpit display design.
\end{abstract}

Keywords: Ergonomic evaluation, intuitionistic fuzzy, ordered weighted geometric average operator, possible degree function.

Received July 12, 2019; accepted September 27, 2020

https://doi.org/10.34028/iajit/18/6/13

\section{Introduction}

The aircraft cockpit display interface is the main source for pilots to obtain the required information. It is required that the information can be transmitted to the pilots accurately to the maximum extent, so as to optimize the decision-making and control, and avoid the occurrence of misjudgment and misoperation accidents [21]. Therefore, the scientific and reasonable design of the man-machine interface plays a very important role in improving the flight efficiency of pilots, reducing pilots' workload, and ensuring flight safety [29].

The ergonomic evaluation of aircraft cockpit displays is an effective means to make aircraft have a good man-machine display interface. The defects of the man-machine display interface design can be found, and the design can be optimized and improved through the evaluation. Many countries have long recognized the importance of aircraft cockpit display ergonomic evaluation, and put great energy into this research field, promoting the rapid development of ergonomic science [6, 14]. These evaluations usually rely on the subjective feelings or experience of aviation experts. Ergonomic evaluation can be quantified by NASA-TLX [18], analytic hierarchy process [23, 27], fuzzy analytic hierarchy process [26], grey area correlation analysis [7], fuzzy comprehensive evaluation method $[1,16]$, etc., These evaluation methods have made a lot of useful achievements in the improvement of evaluation index, weight, and comprehensive evaluation [25]. Compared with the deterministic analytic hierarchy process, it can make the decision results more reasonable. However, due to the complexity of the aircraft cockpit display, the decision-maker cannot give very deterministic preference information for each factor in the decisionmaking process, and there is often some uncertainty, so that the decision-making results are presented as positive, negative, or hesitation between positive and negative. Since the traditional fuzzy set theory can only express the decision-makers' preference information of affirmative and negative, it is increasingly restricted and challenged in its application [12].

Intuitionistic Fuzzy Set (IFS) evaluation method takes full consideration of the positive, negative, and hesitation attitudes in the evaluation through the three dimensions of membership, non-membership, and hesitation [2,3], and aggregates the evaluation value of each index by information integration method, making the evaluation result more reasonable [24, 30]. In view of this, a new intuitionistic fuzzy decision-making approach is proposed to evaluate the cockpit display ergonomic in this study. Firstly, the evaluation index system is established to comprehensively reflect all aspects of the ergonomic characteristics of aircraft cockpit display interface by the Modified Delphi method, and the intuitionistic fuzzy evaluation matrix 
is constructed according to the evaluation index system and the hesitation degree of the evaluators. Secondly, the evaluation matrix is aggregated by using the proposed Intuitionistic Fuzzy Ordered Weighted Geometric Average (IFOWGA) operator. On the basis of the above, the improving possible degree ranking function is used to rank the aggregation values to obtain the evaluation results. Finally, the evaluation method proposed in this paper is used to assess the cockpit display ergonomic of a certain type of aircraft.

\section{Construction of Evaluation Index System}

According to the characteristics of many qualitative studies and limited research data, this work adopts the method to construct the evaluation index system of the cockpit display ergonomic by the Modified Delphi (MD) method [13]. The effective evaluation index system is determined. As shown in Table 1.

Suppose the domain $X$ of IFS is the target layer, and the criterion layer in Table 1 is defined as:

$$
U=\left\{U_{1}, U_{2}, \ldots U_{i} \ldots U_{4}\right\}
$$

For each subclass $U_{i}$ of the criterion layer, the corresponding evaluation index of the index layer is defined as:

$U_{i}=\left\{U_{i 1}, U_{i 2}, U_{i j}, \ldots, U_{i N i}\right\}, i=1, \ldots, 4, N_{i}$ is the number of the index in the criterion layer $U_{i}$.

Table 1. Evaluation index system of cockpit display ergonomic.

\begin{tabular}{|c|c|c|}
\hline Target layer $(U)$ & Criterion layer $\left(U_{i}\right)$ & Index layer $\left(U_{i j}\right)$ \\
\hline \multirow{15}{*}{$\begin{array}{c}\text { Evaluation index } \\
\text { of cockpit display } \\
\text { ergonomic }(U)\end{array}$} & \multirow{5}{*}{ Display contents $\left(U_{1}\right)$} & Character $\left(U_{11}\right)$ \\
\hline & & Brightness $\left(U_{12}\right)$ \\
\hline & & Color $\left(U_{13}\right)$ \\
\hline & & Code $\left(U_{14}\right)$ \\
\hline & & Glare $\left(U_{15}\right)$ \\
\hline & \multirow{4}{*}{$\begin{array}{c}\text { Display characteristics } \\
\qquad\left(U_{2}\right)\end{array}$} & Information readability $\left(U_{21}\right)$ \\
\hline & & Design consistency $\left(U_{22}\right.$ ) \\
\hline & & Information clarity $\left(U_{23}\right)$ \\
\hline & & Function suitability ( $U_{24}$ ) \\
\hline & \multirow{3}{*}{ Reliability $\left(U_{3}\right)$} & Error proofing design $\left(U_{31}\right)$ \\
\hline & & Anti-interference ( $U_{32}$ ) \\
\hline & & Redundancy ( $U_{33}$ ) \\
\hline & \multirow{3}{*}{$\begin{array}{l}\text { Display environment } \\
\qquad\left(U_{4}\right)\end{array}$} & Lighting $\left(U_{41}\right)$ \\
\hline & & Vibration $\left(U_{42}\right)$ \\
\hline & & Thermal environment $\left(U_{43}\right)$ \\
\hline
\end{tabular}

\section{Classification of Overall Ergonomic Evaluation Level}

The classification of the overall ergonomic evaluation level of the aircraft cockpit display should be established according to the characteristics of the ergonomic index of the cockpit display and the comparison of corresponding index benchmarks. The evaluation level is divided into six levels in this work [17], that is, $V=\left\{v_{1}, v_{1}, \ldots, v_{6}\right\}$. Where, $v_{1}$ corresponds to the highest level of ergonomic and $v_{6}$ to the lowest. Each level is described by the meaning of comments, which is more conducive to understanding and practice. The meanings of each level are shown in Table 2.

Table 2. Aircraft cockpit display overall ergonomic level and its meanings.

\begin{tabular}{|c|c|}
\hline Level & Evaluation level meanings \\
\hline$v_{1}$ & $\begin{array}{c}\text { Satisfaction. No compensation from pilots is required to achieve } \\
\text { satisfactory performance. }\end{array}$ \\
\hline$v_{2}$ & $\begin{array}{c}\text { There are negligible defects and no compensation from pilots is } \\
\text { required to achieve satisfactory performance. }\end{array}$ \\
\hline$v_{3}$ & $\begin{array}{c}\text { There are some moderate defects, and appropriate compensation } \\
\text { is required from pilots to achieve sufficient performance. }\end{array}$ \\
\hline$v_{4}$ & $\begin{array}{c}\text { There are some defects with high dissatisfaction, and large } \\
\text { compensation is required from pilots to achieve sufficient } \\
\text { performance. }\end{array}$ \\
\hline$v_{5}$ & $\begin{array}{c}\text { There are some defects with very high dissatisfaction, and strong } \\
\text { compensation is required from pilots to achieve sufficient } \\
\text { performance. }\end{array}$ \\
\hline$v_{6}$ & $\begin{array}{c}\text { There are some large defects, and some of the required } \\
\text { operations cannot be completed. }\end{array}$ \\
\hline
\end{tabular}

\section{Preliminaries: Intuitionistic Fuzzy Sets}

Fuzzy set theory has been widely applied in many fields since it was proposed by Zadeh [31]. IFS proposed by Atanassov [2], which is different from the traditional fuzzy set, measures information more accurately due to fully considering some information of the fuzzy value of membership, nonmembership, and hesitation [22]. Therefore, the method and application based on intuitionistic fuzzy set have strong rationality [10]. Many scholars have carried out in-depth research on IFS, and the representative fuzzy sets include interval fuzzy set [15], intuitionistic fuzzy set [20], interval intuitionistic fuzzy set [33], intuitionistic trapezoidal fuzzy set [8], and hesitative fuzzy set. etc., [11]. The relevant theories and applications of intuitionistic fuzzy set have always been the research hotspots in the fuzzy field.

The intuitionistic fuzzy evaluation is a method of comprehensive evaluation based on fuzzy mathematics by applying the IFS and the synthesis principle of intuitionistic fuzzy relations to quantify some factors with unclear boundaries and difficult quantification [9].

- Definition 1. IFS Let $X$ be a given set. An IFS in $X$ is an object $A$ given by:

$$
A=\left\{\left\langle x, \mu_{A}(x), \gamma_{A}(x)\right\rangle \mid x \in X\right\}
$$

Where $\mu_{A}: X \rightarrow[0,1]$ and $\gamma_{A}: X \rightarrow[0,1]$ represent the membership function and non-membership function of $A$ respectively. And $\forall x \in X$ in $A$, satisfy $0 \leq \mu_{A}(x)+\gamma_{A}(x) \leq 1$. If $\mu_{A}(x)+\gamma_{A}(x)=1, A$ is a common fuzzy set. IFS $A$ can be simplified as $A=\left\langle x, \mu_{A}, \gamma_{A}\right\rangle$ or $A=\left\langle\mu_{A}, \gamma_{A}\right\rangle / x$. The membership 
boundary value of element $x$ in set $A$ is defined as subintervals $\left[\mu_{A}(x), 1-\gamma_{A}(x)\right]$ on $[0,1]$, which is called the intuitionistic fuzzy value of $A$ briefly.

\section{Intuitionistic Fuzzy Evaluation}

\subsection{Construction of Intuitionistic Fuzzy Evaluation Matrixin Criterion Layer}

The process of constructing an intuitionistic fuzzy evaluation matrix is as follows.

Firstly, the evaluation result set $Z$ corresponding to the subclass $U_{i}$ of the criterion layer is defined as:

$$
Z=\left\{z_{1}, z_{2}, \cdots, z_{m}, \cdots, z_{K}\right\}, m=1,2, \ldots, K
$$

Where $K$ represents the number of elements in the evaluation result set. $K$ value can be different from the subclass of evaluation index in the criterion layer, and generally, the same value is adopted. For example, the following evaluation result set is taken in this work, namely: \{excellent, good, average, fair, poor, fail $\}$.

Secondly, several flight experts (Suppose the number of $L$ ) who are experienced and familiar with background make simulation flight on simulator respectively. After the flight, the flight parameters recorded by the computer system are provided to the experts. According to flight parameters and their respective experience, experts determine the probability that each evaluation index $U_{i j}$ belongs to the evaluation level $z_{m}(m=1,2, \ldots, 6) \quad$ (including "belonging to", "not belonging to", and "uncertain" of three cases). The number of $L$ experts who select each case respectively is counted (suppose $R_{i j m}, R_{i j m y}$ and $R_{i j m h}$ ), and the intuitionistic fuzzy evaluation value $b_{i j m}$ that the evaluation index $U_{i j}$ is determined to level $z_{m}(m=1,2, \ldots, 6)$ is given by:

$$
b_{i j m}=\left\langle\frac{R_{i j m \mu}}{L}, \frac{R_{i j m \gamma}}{L}\right\rangle=\left\langle\mu_{i j m}, \gamma_{i j m}\right\rangle
$$

Similarly, intuitionistic fuzzy evaluation values of other indexes can be obtained according to Equation (1), and the intuitionistic fuzzy evaluation matrix of subclass $U_{\mathrm{i}}$ in the criterion layer composed of these values as elements is given by:

$$
B_{i}=\left[\begin{array}{cccc}
b_{i 11} & b_{i 21} & \cdots & b_{i N_{i} 1} \\
b_{i 12} & b_{i 22} & \cdots & b_{i N_{i} 2} \\
\vdots & \vdots & \vdots & \vdots \\
b_{i 16} & b_{i 26} & \cdots & b_{i N_{i} 6}
\end{array}\right]
$$

Equation (2) is the evaluation matrix represented by the intuitionistic fuzzy set, which is converted into the form of intuitionistic fuzzy value as:

$$
\begin{aligned}
\tilde{B}_{i} & =\left\langle\mu_{i j m}, 1-\gamma_{i j m}\right\rangle_{6 \times N_{i}} \\
& =\left[\begin{array}{cccc}
\left\langle\mu_{i 1}, 1-\gamma_{i 11}\right\rangle & \left\langle\mu_{i 21}, 1-\gamma_{i 21}\right\rangle & \cdots & \left\langle\mu_{i i_{i} 1}, 1-\gamma_{i N_{1} i}\right\rangle \\
\left\langle\mu_{i 12}, 1-\gamma_{i 12}\right\rangle & \left\langle\mu_{i 22}, 1-\gamma_{i 22}\right\rangle & \cdots & \left\langle\mu_{i N_{i}}, 1-\gamma_{i N_{i}}\right\rangle \\
\vdots & \vdots & \vdots & \vdots \\
\left\langle\mu_{i 16}, 1-\gamma_{i 16}\right\rangle & \left\langle\mu_{i 26}, 1-\gamma_{i 26}\right\rangle & \cdots & \left\langle\mu_{i N_{i},}, 1-\gamma_{i i_{i} 6}\right\rangle
\end{array}\right]
\end{aligned}
$$

Where, the intuitionistic fuzzy evaluation matrix $\tilde{B}_{i}$ represents the evaluation results of experts on all indexes of subclass $U_{\mathrm{i}}$ in the criterion layer, and it includes all the information of affirmation, negation, and hesitation in expert evaluation. So it makes the evaluation results more accurate.

\subsection{Intuitionistic Fuzzy Information Aggregation}

In order to complete the intuitionistic fuzzy evaluation of the criterion layer, Equation (3) needs to be effectively aggregated and evaluated. For this reason, the basic algorithm of intuitionistic fuzzy is defined in this work, and the operator of Ordered Weighted Geometric Average (OWGA) is extended to the field of IFS based on this algorithm, as well as the operator of IFOWGA operator can be obtained [5]. Next, the basic operation laws of intuitionistic fuzzy value are defined.

- Definition 2 (Basic operations of intuitionistic fuzzy value) Let $\tilde{c}=\left[\mu_{c}, 1-\gamma_{c}\right]$ and $\tilde{d}=\left[\mu_{d}, 1-\gamma_{d}\right]$ be intuitionistic Fuzzy Values, and basic operation laws are defined as follows:

1. $\tilde{e}=\tilde{c} \otimes \tilde{d}=\left[\mu_{c} \mu_{d},\left(1-\gamma_{c}\right)\left(1-\gamma_{d}\right)\right]$;

2. $\tilde{f}=\tilde{c}^{\lambda}=\left[\mu_{c}^{\lambda},\left(1-\gamma_{c}\right)^{\lambda}\right], \lambda>0$.

Obviously, $\tilde{e}$ and $\tilde{f}$ are still intuitionistic fuzzy values. According to the operation law of definition 2, the OWGA operator is extended to IFS.

- Definition 3 IFOWGA operator Let $\left[\tilde{c}_{1}, \tilde{c}_{2}, \cdots, \tilde{c}_{n}\right]$ be a group of intuitionistic fuzzy values, and $P: T^{n} \rightarrow$ $T$.

$$
P\left(\tilde{c}_{1}, \tilde{c}_{2}, \cdots, \tilde{c}_{n}\right)=\prod_{j=1}^{n} \tilde{d}_{j}^{\omega_{j}}==\left(\tilde{d}_{1}\right)^{\omega_{1}} \otimes\left(\tilde{d}_{2}\right)^{\omega_{2}} \otimes \cdots \otimes\left(\tilde{d}_{n}\right)^{\omega_{n}}
$$

Where $\tilde{d}_{j}$ is the $j$ th largest element in $\left[\tilde{c}_{1}, \tilde{c}_{2}, \cdots, \tilde{c}_{n}\right]$; $W=\left(\omega_{1}, \omega_{2}, \ldots, \omega_{n}\right)$ is the n-dimensional weighted vector associated with $P, \omega_{j} \in[0,1], 1 \leq j \leq n$, and $\sum_{j=1}^{n} \omega_{j}=1$. So $P$ is the n-dimensional IFOWGA operator.

Next, the intuitionistic fuzzy aggregation of the criterion layer is illustrated by taking the subclass $U_{1}$ as an example. For the weighted vectors of IFOWGA operator in Equation (4), Xu and Yager [28] proposed a 
weighting method based on normal distribution, which has rich properties. In this work, according to the index number of subclass $U_{1}$ in the criterion layer, the weighted vector is assumed as:

$$
\omega=\left(\omega_{1}, \omega_{2}, \omega_{3}, \omega_{4}, \omega_{5}\right)
$$

According to the IFOWGA operator provided by Equation (4), the matrix $\tilde{B}_{1}$ in Equation (3) is aggregated, and $U_{1}$ is determined to be the intuitionistic fuzzy aggregated value of each evaluation level $z_{m}(m=1,2, \ldots, 6)$, which can be given by:

$$
\begin{aligned}
& \tilde{z}_{1 m}=\prod_{j=1}^{5}\left[\left\langle\mu_{1 j m}, 1-\gamma_{1 j m}\right\rangle\right]^{\omega_{j}}= \\
& {\left[\left\langle\mu_{11 m}, 1-\gamma_{11 m}\right\rangle\right]^{\omega_{1}} \otimes\left[\left\langle\mu_{12 m}, 1-\gamma_{12 m}\right\rangle\right]^{\omega_{2}} \otimes} \\
& {\left[\left\langle\mu_{13 m}, 1-\gamma_{13 m}\right\rangle\right]^{\omega_{3}} \otimes\left[\left\langle\mu_{14 m}, 1-\gamma_{14 m}\right\rangle\right]^{\omega_{4}} \otimes} \\
& {\left[\left\langle\mu_{15 m}, 1-\gamma_{15 m}\right\rangle\right]^{\omega_{5}}}
\end{aligned}
$$

Where $\tilde{z}_{1 m}(m=1,2, \ldots, 6)$ indicates that subclass $U_{1}$ of the criterion layer is determined as the membership degree, non-membership degree, and hesitation degree of each evaluation level $z_{m}(m=1,2, \ldots, 6)$. Similarly, according to Equations (1) to (6), the intuitionistic fuzzy value that each subclasses $U_{i}(i=1,2, \ldots, 4)$ is determined to each level $z_{m}(m=1,2, \ldots, 6) \quad$ is given by $\tilde{z}_{\text {im }}(i=1,2, \ldots, 4 . m=1,2, \ldots, 6)$.

Then, the evaluation matrix composed of $\tilde{z}_{\text {iim }}(i=1,2, \ldots, 4 . m=1,2, \ldots, 6)$ as elements in the target layer is constructed as:

$$
\tilde{B}=\left[\begin{array}{cccc}
\tilde{z}_{11} & \tilde{z}_{21} & \cdots & \tilde{z}_{41} \\
\tilde{z}_{12} & \tilde{z}_{22} & \cdots & \tilde{z}_{42} \\
\vdots & \vdots & \vdots & \vdots \\
\tilde{z}_{16} & \tilde{z}_{26} & \cdots & \tilde{z}_{46}
\end{array}\right]
$$

According to the IFOWGA operator provided by Equation (4), the matrix $\tilde{B}$ in Equation (7) is aggregated, and the intuitionistic fuzzy evaluation value that $U$ is determined to each level $z_{m}(m=1,2, \ldots, 6)$ is acquired as $\tilde{z}_{m}(m=1,2, \cdots, 6) . \quad \tilde{z}_{m}(m=1,2, \cdots, 6)$ represents that the cockpit display ergonomic belongs to the membership degree, non-membership degree, and hesitation degree of each evaluation level. What level the ergonomic evaluation belongs to needs to be further processed for the above six intuitionistic fuzzy values.

\subsection{Synthetic Evaluation based on Improving Possible Degree Function}

In order to get the final evaluation results, the above intuitionistic fuzzy values need to be synthesized, so the possible degree function is introduced to compare and rank them. In this section, the possibility degree between intuitionistic fuzzy sets is firstly defined, and the intuitionistic fuzzy value ranking method based on the improving possible degree function is proposed to rank the intuitionistic fuzzy value of evaluation level. The possible degree reflecting the magnitude relationship between intuitionistic fuzzy sets is defined as follows.

- Definition 4 (Possible degree) $\forall A, B \in \operatorname{IFS}(X)$, if the function $p(A \geq B)$ satisfies:

1. $0 \leq p(A \geq B) \leq 1$

2. If and only if $1-\gamma_{B}(x) \leq \mu_{A}(x), p(A \geq B)=1$

3. If and only if $1-\mu_{B}(x) \leq \gamma_{A}(x), p(A \geq B)=0$

4. $p(A \geq B)+p(B \geq A)=1, \quad$ in particular $p(A \geq B)=1 / 2$

5. If and only if $\mu_{A}(x)-\gamma_{A}(x) \geq \mu_{B}(x)-\gamma_{B}(x)$, $p(A \geq B) \geq 1 / 2$

6. $\forall C \in I F S(X)$, if $p(A \geq B) \geq 1 / 2 \quad$ and $p(B \geq C) \geq 1 / 2$, then $p(A \geq C) \geq 1 / 2$.

Then, function $p(A \geq B)$ is called the possible degree of IFS [19].

In order to extend the possible degree function to IFS, the favorite possible degree measure formula is given by theorem 1 below.

- Theorem 1 Suppose $\forall A, B \in I F S(x)$, then

$$
p(A \geq B)=\max \left\{1-\max \left\{\frac{1-\gamma_{B}(x)-\mu_{A}(x)}{\pi_{A}(x)+\pi_{B}(x)}, 0\right\}, 0\right\}
$$

Equation (8) is called the possible degree of $A \geq B$.

The proof of above theorem is similar to that on the real number set [4], so it is ignored here.

For $\tilde{z}_{m}(m=1,2, \cdots, 6)$, according to Equation (8) the possible degree matrix is given as follows by comparing them in pairs.

$$
S=\left[\begin{array}{cccc}
0.5 & p_{12} & \cdots & p_{16} \\
p_{21} & 0.5 & \cdots & p_{26} \\
\vdots & \vdots & \ddots & \vdots \\
p_{61} & p_{62} & \cdots & 0.5
\end{array}\right]
$$

Equation (9) is a fuzzy complementary judgment matrix, and the order of all intuitionistic fuzzy values can be given by the transitivity of a possible degree. In this work, the ranking equation of intuitionistic fuzzy values are given as follows [32].

$$
\rho_{m}=\frac{1}{N(N-1)}\left(\sum_{n=1}^{N} p_{m n}+\frac{N}{2}-1\right)
$$

According to Equation (10), the ranking vector $\rho=$ $\left(\rho_{1}, \rho_{2}, \ldots, \rho_{6}\right)$ of matrix $S$ can be obtained, and the 
intuitionistic fuzzy values $\tilde{z}_{m}(m=1,2, \cdots, 6)$ are ordered according to the magnitude of $\rho_{m}$. If the following equation is satisfied,

$$
\rho_{m^{\prime}}=\max _{1 \leq m \leq 6}\left(\rho_{m}\right)
$$

Then for the index $U$ of the overall target layer, the result of the intuitionistic fuzzy evaluation is $\tilde{z}_{m^{\prime}}$, and the corresponding evaluation result is level $v_{m^{\prime}}$.

\section{Example and Simulation}

\subsection{Example Application}

According to the evaluation approach proposed in this work, an evaluation example of the cockpit display ergonomic is conducted by 24 experienced active pilots based on a certain aircraft simulator. The display screen and logic of the cockpit display system are consistent with the real one, which can simulate the flight state in real time, and the equipment display changes and switches in real-time with flight changing. Before evaluation, 24 pilots need to accomplish a whole dynamic flight process in a flight simulator, which mainly includes cruise tasks, taking-off, and landing, etc. The flight simulation time is about 830 seconds due to slight variations in the operating time of different pilots when they perform manual take-off or landing operations. In the process of flight simulation, the computer system automatically records flight parameters, which are provided to the pilot to assist in decision-making after the flight. The recorded flight parameters mainly include aircraft pitch Angle, airspeed, air pressure height, course Angle and roll Angle, etc. The specific steps of ergonomic evaluation are as follows.

- Step 1: According to the evaluation index system established in Table 1 and Equation (2), the intuitionistic fuzzy evaluation matrix composed of $b_{i j m}$ as elements in the criterion layer is formed in Table 3.

Table 3. Intuitionistic fuzzy evaluation matrix of the criterion layer.

\begin{tabular}{|c|c|c|c|c|c|c|c|}
\hline \multicolumn{2}{|c|}{ level } & $z_{1}$ & $z_{2}$ & $z_{3}$ & $z_{4}$ & $z_{5}$ & $z_{6}$ \\
\hline \multirow{5}{*}{$U_{1}$} & $U_{11}$ & $\langle 0.50,0.29\rangle$ & $\langle 0.92,0.04\rangle$ & $\langle 0.42,0.50\rangle$ & $\langle 0.33,0.54\rangle$ & $\langle 0.21,0.67\rangle$ & $\langle 0.13,0.71\rangle$ \\
\hline & $U_{12}$ & $\mid\langle 0.25,0.67\rangle$ & $\langle 0.75,0.25\rangle$ & $\langle 0.58,0.38\rangle$ & $\langle 0.46,0.50\rangle$ & $\langle 0.38,0.50\rangle$ & $\langle 0.29,0.63\rangle$ \\
\hline & $U_{13}$ & $\langle 0.54,0.25\rangle$ & $\langle 0.50,0.42\rangle$ & $\langle 0.67,0.25\rangle$ & $\langle 0.42,0.54\rangle$ & $\langle 0.42,0.46\rangle$ & $\mid\langle 0.33,0.67\rangle$ \\
\hline & $U_{14}$ & $\langle 0.42,0.38\rangle$ & $\langle 0.83,0.08\rangle$ & $\langle 0.46,0.46\rangle$ & $\langle 0.38,0.54\rangle$ & $\langle 0.25,0.71\rangle$ & $\langle 0.17,0.79\rangle$ \\
\hline & $U_{15}$ & $\langle 0.71,0.17\rangle$ & $\langle 0.58,0.42\rangle$ & $\langle 0.46,0.42\rangle$ & $\langle 0.33,0.50\rangle$ & $\langle 0.38,0.54\rangle$ & $\langle 0.29,0.67\rangle$ \\
\hline \multirow{4}{*}{$U_{2}$} & $U_{21}$ & $\langle 0.42,0.54\rangle$ & $\langle 0.67,0.29\rangle$ & $\langle 0.29,0.58\rangle$ & $\langle 0.21,0.71\rangle$ & $\langle 0.17,0.79\rangle$ & $\langle 0.13,0.83\rangle$ \\
\hline & $U_{22}$ & $\langle 0.25,0.67\rangle$ & $\langle 0.96,0.04\rangle$ & $\langle 0.17,0.75\rangle$ & $\langle 0.08,0.83\rangle$ & $\langle 0.13,0.71\rangle$ & $\langle 0.04,0.92\rangle$ \\
\hline & $U_{23}$ & $\langle 0.21,0.71\rangle$ & $\langle 0.88,0.08\rangle$ & $\langle 0.33,0.54\rangle$ & $\langle 0.17,0.67\rangle$ & $\langle 0.13,0.79\rangle$ & $\langle 0.08,0.92\rangle$ \\
\hline & $U_{24}$ & $\langle 0.38,0.50\rangle$ & $\langle 0.58,0.25\rangle$ & $\langle 0.25,0.54\rangle$ & $\langle 0.53,0.33\rangle$ & $\langle 0.21,0.71\rangle$ & $\langle 0.17,0.75\rangle$ \\
\hline \multirow{3}{*}{$U_{3}$} & $U_{31}$ & $\mid\langle 0.29,0.67\rangle$ & $\langle 0.88,0.04\rangle$ & $\langle 0.29,0.54\rangle$ & $\langle 0.21,0.67\rangle$ & $\langle 0.17,0.75\rangle$ & $\langle 0.08,0.75\rangle$ \\
\hline & $U_{32}$ & $\langle 0.33,0.54\rangle$ & $\langle 0.67,0.17\rangle$ & $\langle 0.46,0.42\rangle$ & $\langle 0.21,0.63\rangle$ & $\langle 0.33,0.50\rangle$ & $\langle 0.25,0.58\rangle$ \\
\hline & $U_{33}$ & $\langle 0.33,0.63\rangle$ & $\langle 0.75,0.21\rangle$ & $\langle 0.38,0.54\rangle$ & $\langle 0.17,0.75\rangle$ & $\langle 0.21,0.63\rangle$ & $\langle 0.04,0.92\rangle$ \\
\hline \multirow{3}{*}{$U_{4}$} & $U_{41}$ & $\langle 0.38,0.46\rangle$ & $\langle 0.25,0.71\rangle$ & $\langle 0.33,0.67\rangle$ & $\langle 0.71,0.13\rangle$ & $\langle 0.17,0.75\rangle$ & $\langle 0.08,0.83\rangle$ \\
\hline & $U_{42}$ & $\langle 0.21,0.75\rangle$ & $\langle 0.13,0.83\rangle$ & $\langle 0.17,0.79\rangle$ & $\langle 0.92,0.04\rangle$ & $\langle 0.08,0.88\rangle$ & $\langle 0.04,0.92\rangle$ \\
\hline & $U_{43}$ & $\langle 0.29,0.58\rangle$ & $\langle 0.21,0.67\rangle$ & $\langle 0.29,0.54\rangle$ & $\langle 0.83,0.13\rangle$ & $\langle 0.25,0.67\rangle$ & $\langle 0.08,0.88\rangle$ \\
\hline
\end{tabular}

The specific calculation method of element in Table 3 is to count the numbers of pilots who respectively choose membership and nonmembership for each index and they are applied to the Equation (1). Then the matrix provided by Table 3 is constructed by the calculation result.

- Step 2: According to the IFOWGA aggregation operator provided by Equation (4), the intuitionistic fuzzy evaluation matrix of the criterion layer obtained in the previous step is aggregated to get the intuitionistic fuzzy value $\tilde{z}_{\text {im }}(i=1,2, \ldots, 4 . m=1,2, \ldots, 6)$, and the intuitionistic fuzzy evaluation matrix of the target layer is constructed with $\tilde{z}_{i m}$ as elements, as shown in Table 4.

Table 4. Intuitionistic fuzzy evaluation matrix of the target layer.

\begin{tabular}{|c|c|c|c|c|c|c|}
\hline level & $z_{1}$ & $z_{2}$ & $z_{3}$ & $z_{4}$ & $z_{5}$ & $z_{6}$ \\
\hline$U_{1}$ & $\langle 0.49,0.53\rangle$ & $\langle 0.72,0.76\rangle$ & $\langle 0.47,0.49\rangle$ & $\langle 0.38,0.43\rangle$ & $\langle 0.33,0.38\rangle$ & $\langle 0.24,0.29\rangle$ \\
\hline$U_{2}$ & $\langle 0.32,0.36\rangle$ & $\langle 0.77,0.82\rangle$ & $\langle 0.26,0.31\rangle$ & $\langle 0.24,0.34\rangle$ & $\langle 0.16,0.25\rangle$ & $\langle 0.10,0.14\rangle$ \\
\hline$U_{3}$ & $\langle 0.39,0.42\rangle$ & $\langle 0.78,0.84\rangle$ & $<0.38,0.42>$ & $\langle 0.23,0.27\rangle$ & $\langle 0.21,0.29\rangle$ & $\langle 0.12,0.19\rangle$ \\
\hline$U_{4}$ & $\langle 0.29,0.33\rangle$ & $\langle 0.32,0.40\rangle$ & $\langle 0.28,0.31\rangle$ & $\langle 0.65,0.70\rangle$ & $\langle 0.17,0.25\rangle$ & $\langle 0.07,0.11\rangle$ \\
\hline
\end{tabular}

The elements in Table 4 are calculated by applying the weight coefficient and the elements in each row of Table 3 to Equation (6). Then the matrix provided by Table 4 is constructed by the calculation result.

- Step 3: IFOWGA operator is used to aggregate the intuitionistic fuzzy evaluation matrix of the target layer, and the intuitionistic fuzzy values that the target layer $U$ is judged as each level $Z_{\mathrm{m}}(m=1,2, \ldots, 6)$ are given by:

$$
\begin{aligned}
& \tilde{z}_{1}=\langle 0.3819,0.4041\rangle, \tilde{z}_{2}=\langle 0.7056,0.7341\rangle, \\
& \tilde{z}_{3}=\langle 0.3475,0.3750\rangle, \tilde{z}_{4}=\langle 0.3635,0.4022\rangle,
\end{aligned}
$$




$$
\tilde{z}_{5}=\langle 0.2175,0.2425\rangle, \tilde{z}_{6}=\langle 0.1325,0.1615\rangle
$$

- Step 4: According to the possible degree function defined by Equation (8), $\quad \tilde{z}_{m}(m=1,2, \ldots, 6)$ are compared in pairs to obtain the measure $p$ between two intuitionistic fuzzy values, and through Equation (9) the possible degree matrix is given by:

$$
S=\left[\begin{array}{cccccc}
0.5 & 0 & 1 & 1 & 1 & 1 \\
1 & 0.5 & 1 & 1 & 1 & 1 \\
0 & 0 & 0.5 & 1 & 1 & 1 \\
0 & 0 & 0 & 0.5 & 0.862 & 1 \\
0 & 0 & 0 & 0.138 & 0.5 & 1 \\
0 & 0 & 0 & 0 & 0 & 0.5
\end{array}\right]
$$

According to the ranking formula provided by Equation (10), the ranking vector of the above matrix is calculated as: $\rho=\left(\begin{array}{llll}0.2167 & 0.2500 & 0.1833 & 0.1454\end{array}\right.$ 0.12130 .0833 )

According to the ranking vector, the ranking of the intuitionistic fuzzy values $\tilde{z}_{m}(m=1,2, \cdots, 6)$ is obtained, and the ranking results are shown in Table 5.

Table 5. Comprehensive ranking of different evaluation levels.

\begin{tabular}{|c|c|c|c|c|}
\hline SN & Level & Value & Ranking Vector & Ranking \\
\hline 1 & $v_{1}$ & $\langle 0.3819,0.4041\rangle$ & 0.2167 & 2 \\
\hline 2 & $v_{2}$ & $\langle 0.7056,0.7341\rangle$ & 0.2500 & 1 \\
\hline 3 & $v_{3}$ & $\langle 0.3474,0.3750\rangle$ & 0.1833 & 3 \\
\hline 4 & $v_{4}$ & $\langle 0.3635,0.4022\rangle$ & 0.1454 & 4 \\
\hline 5 & $v_{5}$ & $\langle 0.2172,0.2425\rangle$ & 0.1213 & 5 \\
\hline 6 & $v_{6}$ & $\langle 0.1326,0.1618\rangle$ & 0.0833 & 6 \\
\hline
\end{tabular}

The ranking of evaluation level in Table 5 is carried out according to values in the ranking vector.

Since $\rho_{2}=\max _{1 \leq m \leq 6}\left(\rho_{m}\right)$, the final intuitionistic fuzzy evaluation value is $\tilde{z}_{m^{\prime}}=\tilde{z}_{2}$, and the ergonomic level of the cockpit display of this type of aircraft is " $v_{2}$ ". It can be seen from the analysis of the intuitionistic fuzzy evaluation matrix of the target layer that the aircraft cockpit display ergonomic in the aspect of subclasses $U_{1}, U_{2}$, and $U_{3}$ of the criterion layer is good, but the ergonomic evaluation in the aspect of $U_{4}$ is poorer, namely, the pilots think the aircraft cockpit display levels in the aspects of "display contents", "display characteristics" and "reliability" are "good", but in the aspect of "display environment" is "poor". So the design level of the cockpit display environment still needs to be further improved.

\subsection{Simulation and Analysis}

In order to examine the stability of the evaluation result of intuitionistic fuzzy, the intuitionistic fuzzy values $\tilde{z}_{m^{\prime}}$ calculated based on the different number of experts are compared and analyzed, and the changing trend of the intuitionistic fuzzy value $\tilde{z}_{m^{\prime}}$ with the increasing number of experts is shown in Figure 3.

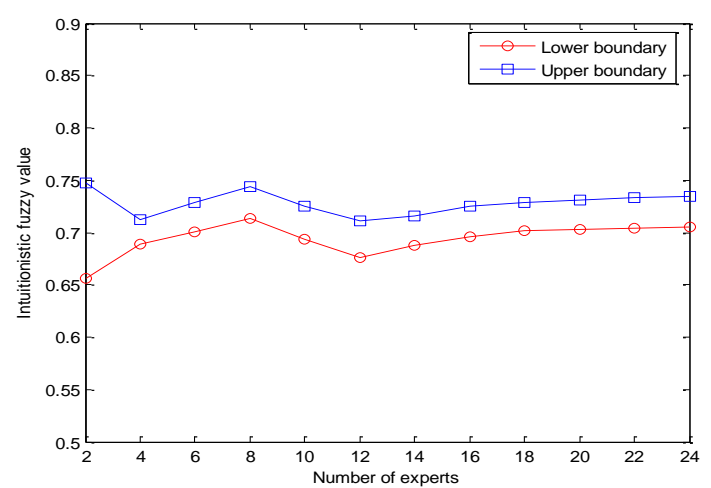

Figure 1. Comparison of intuitionistic fuzzy values $\tilde{z}_{m^{\prime}}$ for different expert numbers.

As can be seen from Figure 1, when the number of experts is more than 16 , the variation of intuitionistic fuzzy value is already small. And when the number of experts is more than 22 , the calculated results are stable. It can be shown that the intuitionistic fuzzy evaluation method adopted in this work has good stability.

\section{Conclusions}

In this work, the key problems in the ergonomic evaluation of aircraft cockpit displays are studied, and the evaluation approach based on intuitionistic fuzzy is scientific, rational, and applicable to engineering. The proposed quantitative evaluation approach based on intuitionistic fuzzy describes the fuzziness of expert evaluation through the "non-membership degree" attribute, considers the hesitation degree of expert decision-making, and carries out comprehensive processing of multi-attribute decision-making through IFOWGA operator and possible degree function, so as to realize the quantitative evaluation of aircraft cockpit display ergonomic. It can be shown from the example analysis results that the proposed ergonomic evaluation approach of aircraft cockpit display and data processing method can be applied to the engineering practice, and classify the existing problems in the cockpit. display. The proposed approach can provide some technical references for the man-machine ergonomic evaluation of military aircraft, and also can be further extended to other complex systems.

\section{Acknowledgment}

This work was supported by the National Natural Science Foundation of China (Grant No.61861033), the Science and Technology Project of Jiangxi Education Department (No.DA202104169),China's Jiangxi Province Natural Science Foundation (No. 20202ACBL202007), the Ministry of education of Humanities and Social Science project (No. 20YJC880087) and China Postdoctoral Science Foundation（No. 2019M662259）. 


\section{References}

[1] Alvarez J. and Mercado P., "Online Inference of The Dynamic Security Level of Power Systems Using Fuzzy Techniques," IEEE Transactions on Power Systems, vol. 22, no. 2, pp. 717-726, 2007.

[2] Atanassov K., "Intuitionistic Fuzzy Sets," Fuzzy Sets and Systems, vol. 20, no. 1, pp. 87-96, 1986.

[3] Bustince H. and Burillo P., "Vague Sets are Intuitionistic Fuzzy Sets," Fuzzy Sets and Systems, vol. 79, no. 3, pp. 403-405, 1996.

[4] Chaji A., Fukuyama H., and Shiraz R., "Selecting A Model for Generating OWA Operator Weights in MAGDM Problems by Maximum Entropy Membership Function," Computers and Industrial Engineering, vol. 124, pp. 370-378, 2018.

[5] Chen D. and Zhang L., "Intuitionistic Fuzzy Identification of Air Attack Target Type," Control and Decision, vol. 26, no. 7, pp. 1046-1049, 2011.

[6] Craig J. and Burrett G., "The Design of A Human Factors Questionnaire for Cockpit Assessment," in Proceedings of International Conference on Human Interfaces in Control Rooms, Cockpits and Command Centres, Bath, pp. 16-20, 2002.

[7] Cui M., Sun Y., Yang J., and Weinan W., "Power Grid Security Comprehensive Assessment Based on Multi Level Grey Area Relational Analysis," Power System Technology, vol. 37, no. 12, pp. 345 3-3460, 2013.

[8] Dahiya N., Bhatnagar V., and Singh M., "A Fuzzy Based Matrix Methodology for Evaluation and Ranking of Data Warehouse Conceptual Models Metrics," The International Arab Journal of Information Technology, vol. 15, no. 2, pp. 202-212, 2018.

[9] Das S., Kar S., and Pal T., "Robust Decision Making using Intuitionistic Fuzzy Numbers," Granular Computing, vol. 2, no. 1, pp. 41-54, 2017.

[10] Devi K. and Yadav S., "A Multicriteria Intuitionistic Fuzzy Group Decision Making for Plant Location Selection with ELECTRE Method," International Journal of Advanced Manufacturing Technology, vol. 66, no. 9-12, pp. 1219-1229, 2013.

[11] Garg H. and Kumar K., "Improved Possibility Degree Method for Ranking Intuitionistic Fuzzy Numbers and their Application in Multiattribute Decision-Making," Granular Computing, vol. 4, no. 2, pp. 237-247, 2018.

[12] Geng T., Zhang A., and Lu G., "Consensus Intuitionistic Fuzzy Group Decision-Making Method for Aircraft Cockpit Display and Control System Evaluation," Journal of Systems Engineering and Electronics, vol. 24, no. 4, pp. 634-641, 2013.
[13] Guo Z., Guo D., Yang J., and Huang C., "Study on Evaluation Index System of Helicopter Display Interface Ergonomics," Electronics Optics and Control, vol. 18, no. 5, pp. 68-69, 2011.

[14] Havir T., Durbin D., Frederick L., and Hicks J., "Human Factors Assessment of the UH-60M Crew Station during the Limited User Test," Technical Report, 2006.

[15] He Y., Chen H., Zhou L., Liu J., and Tao Z., "Intuitionistic Fuzzy Geometric Interaction Averaging Operators and their Application to Multi-Criteria Decision Making," Information Sciences, vol. 259, no. 3, pp. 142-159, 2014.

[16] Li L., Yao J., Long L., Yuan X., and Li H., "Application of Combination Weighing Method in Fuzzy Synthetic Evaluation of Power Quality," Automation of Electric Power Systems, vol. 31, no. 4, pp. 56-60, 2007.

[17] Li Y., Yuan X., and Yang F., "Multilevel Fuzzy Evaluation of the Cockpit Ergonomics," China Safety Science Journal, vol. 13, no. 3, pp. 51, 2003.

[18] Liao J., "Mental workload and its Measurement," Journal of System Engineering, vol. 10, no. 3, pp. 1192-1231, 1995.

[19] Liu F., Pedrycz W., and Liu X., "Flexibility Degree of Fuzzy Numbers and its Implication to a Group-Decision-Making Model," IEEE Transactions on Cybernetics, vol. 5, no. 27, pp. 1-12, 2018.

[20] Liu H., Tu J., and Sun C., "Improved Possibility Degree Method for Intuitionistic Fuzzy MultiAttribute Decision Making and Application in Aircraft Cockpit Display Ergonomic Evaluation," IEEE Access, vol. 8, pp. 202540-202554, 2020.

[21] Liu X., "Task-oriented Indicator System for Human Factor Assessment of Fighter Cockpit," Mechanical Engineer, vol. 6, pp. 88-90, 2018.

[22] Maldonado-Macías A., Alvarado A., García J., and Balderrama C., "Intuitionistic fuzzy TOPSIS for Ergonomic Compatibility Evaluation of Advanced Manufacturing Technology," International Journal of Advanced Manufacturing Technology, vol. 70, no. 9-12, pp. 2283-2292, 2014.

[23] Mao A. and He J., "An Approach of Power Grid Security Comprehensive Assessment Based on Credibility Theory," Power System Protection and Control, vol. 39, no. 18, pp. 80-87, 2011.

[24] Tan C. and Chen X., "Generalized Archimedean Intuitionistic Fuzzy Averaging Aggregation Operators and their Application to Multicriteria Decision-Making," International Journal of Information Technology and Decision Making, vol. 15, no. 02, pp. 311-352, 2016.

[25] Wang H., Wu G., and Liu B., "Brief Analysis of Aircraft Cockpit Display/Control System 
Design," Technology and Innovation, vol. 5, pp. 15-16, 2016.

[26] Wang Q., Wen F., Liu M., and Yi S., "Combined Use of Fuzzy Set Theory and Analytic Hierarchy Process for Comprehensive Assessment of Electricity Markets," Automation of Electric Power Systems, vol. 33, no. 7, pp. 32-37, 2009.

[27] Xu P., Yang S., Li F., and Feng S., "Power Grid Security Index Calculation and Display Method Based on AHP and Variable Weight Mechanism," Automation of Electric Power Systems, vol. 39, no. 8, pp. 133-140, 2015.

[28] Xu Z. and Yager R., "Some Geometric Aggregation Operators Based on Intuitionistic Fuzzy Sets," International Journal of General Systems, vol. 35, no. 4, pp. 417-433, 2006.

[29] Xue Y. and Fu G., "Statistical Analysis of the Action Path of Unsafe Act Causes in General Aviation Accidents," Safety and Environmental Engineering, vol. 25, no. 2, pp. 131, 2018.

[30] Yager R., "Prioritized Aggregation Operators," International Journal of Approximate Reasoning, vol. 48, no. 1, pp. 263-274, 2008.

[31] Zadeh L., "Fuzzy Sets," Information and Control, vol. 8, pp. 338-353, 1965.

[32] Zhao H., Xu Z., and Liu S., "Dual Hesitant Fuzzy Information Aggregation with Einstein T-Conorm and T-Norm," Journal of Systems Science and Systems Engineering, vol. 26, no. 2, pp. 240-264, 2017.

[33] Zhou L., Tao Z., and Chen H., "Continuous Interval-Valued Intuitionistic Fuzzy Aggregation Operators and their Applications to Group Decision Making," Applied Mathematical Modelling, vol. 38, no. 7-8, pp. 2190-2205, 2014.

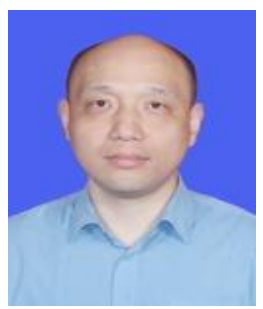

Hui Liu received the B.S. and the M.S. degrees in Communication and Information System from the University of Kongjun Engineering, China, in 1999 and 2003, and the $\mathrm{Ph} . \mathrm{D}$. degree in communication and information system from Kongjun Engineering University in 2006. He is also a Lecture in electronic engineering at information and engineering school for Nanchang Hangkong University, Nanchang, China. His research interestsare in the fields of avonics, intuitionistic fuzzy set theory and ergonomics evaluation.

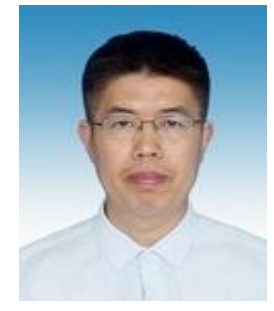

Chengli Sun received the B.S. degree in electronics engineering from Zhongbei University, Taiyuan, China, in 1999, and the Ph.D. degree in signal and information processing from Beijing University of Posts and Telecommunication, Beijing, China. $\mathrm{He}$ is currently a Professor with Information Technology School, Nanchang Hangkong University, China. His current research interests include biomedical image analysis, image processing, speech recognition, speech enhancement, and acoustic event detection.

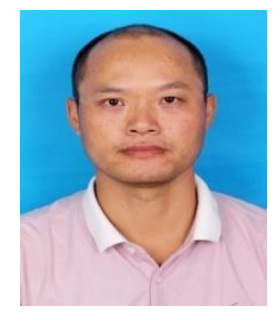

Jiliang Tu received the Master degree of systems engineering from Harbin University of Science and Technology in 2005, Heilongjiang, China and he completed his research Ph.D. of traffic Information Engineering \& Control in Tongji University in 2013, China. He is currently working as an associate Professor (Senior Grade) at School of information and engineering, Nanchang Hangkong University, China. His research is focusing on system engineering theory and practice, equipment supportability theory. 